\title{
ADEQUATE TACIT KNOWLEDGE MANAGEMENT FOR RAILWAYS: A CONCEPTUAL FRAMEWORK
}

\author{
Yawar Abbas \\ Design, Production and Management Department, University of Twente, Enschede, The Netherlands. E-mail: \\ yawar.abbas@utwente.nl \\ Mohammad Rajabalinejad \\ Design, Production and Management Department, University of Twente, Enschede, The Netherlands. E-mail: \\ m.rajabalinejad@utwente.nl
}

Tacit Knowledge (TK) management has been in the limelight for the last few decades with strategic importance for some organizations. Conversion of tacit to explicit knowledge offers a great value to organizations such as Railways in terms of better decision making and minimization of unintended consequences. The challenge, however, comes from the very nature of TK as it cannot be formally communicated and easily converted to explicit knowledge. The paper highlights the significance of TK for Railways specifically in the context of systems integration.

As a research methodology, a brief literature review and interviews at the Netherlands Railways were conducted to identify key features that play a role in TK sharing and its transfer to explicit knowledge. Consequently, a conceptual framework was developed that stresses focusing on four key features for adequate TK management these include context, TK focus, TK constructs, and TK embedding. Moreover, the paper states that the presented approach assists in managing the level of uncertainty related to TK in systems integration context.

The underlying value of this paper is that it presents a testable framework for TK transfer, as it was developed by incorporating the findings from the interviews and literature. Future research stemming from this work is the testing of the framework in a case study and realization of the extent to which it supports a smooth systems integration.

Keywords: tacit knowledge, explicit knowledge, railways, systems integration, knowledge constructs, knowledge management.

\section{Introduction}

The public demand for improved performance of complicated systems like for example the Railways systems has risen significantly in the past few decades. The advancement in technology and innovation has helped the organizations to keep up to the pace of rising public expectations. In the Railways the technology is being used for better maintenance with concepts such as condition-based maintenance and optimization of maintenance plans. Similarly, robust, functional and reliable Safety Management System (SMS) has been developed thanks to better utilization of technology and use of organization's knowledge management. Systems such as Railways are expected to perform safer and be more reliable than ever before. To successfully deliver and live up to these expectations a shift of mindset is required within the organizations. This includes seeing the management of organization's TK as fuel to the optimization of current performances and means of achieving desired levels of integration. Lately, there has a shift in the way the organizations see knowledge with some regarding it as an organizational asset (Kakabadse, Kouzmin, and Kakabadse 2001). Within the knowledge management community there exists a consensus stating that there are two main types of knowledge namely, explicit and tacit. So far in the Railways sector key focus has been on managing explicit knowledge which manifests itself in the form of regulations, manuals and other documents related to structural, functional and operational aspects of the system. In contrast to this, the research on management of TK within the Railways sector is a relatively novel field. Some studies have been conducted on the topic of TK within Railways system which includes the modeling a workspace for TK management by Penciuc, Abel, \& Van Den Abeele, (2010) to improve the Railways system design, and by Siegel \& Schraagen, (2017) to make resilience related knowledge more explicit to name a few. However, improvement of Railways performance on a system level requires a holistic approach towards TK management. Current systems such as SMS, Quality Management System (QMS) and Asset Management System (AMS) fall short in incorporating TK about the use/misuse and malfunction of the system. Besides this, silo mentality within these systems can further exacerbate the situation and result in unintended consequences. Therefore, a holistic approach towards management and dissemination of knowledge in general and TK, in particular, is

Proceedings of the 29th European Safety and Reliability Conference.

Edited by Michael Beer and Enrico Zio

Copyright (c) 2019 European Safety and Reliability Association.

Published by Research Publishing, Singapore.

ISBN: 978-981-11-2724-3; doi:10.3850/978-981-11-2724-3_0837-cd 
required at systems integration level within the Railways system as also stated by Rajabalinejad, (2018b) in his previous works. This paper investigates the $\mathrm{TK}$ required for proper integration of different systems within the Railways and presents to provide a conceptual framework for its management.

\section{State of the art}

As mentioned in the introduction, management of TK within Railways is a novel research field. There have been indications of organizations being concerned about their retiring experienced workforce with some researchers stressing to focus on people and propose enhancing peoples' capabilities through better communication and information transfer (Al-Hawamdeh 2002). The concept of TK is credited to Michael Polayani as he famously argued: "We know more than we can tell" (Polanyi 1966). Within the knowledge management community, the classification of knowledge into tacit and explicit is widely accepted however, conversion of one to the other is highly debated. In simple terms, explicit knowledge is the one that can be easily articulated into different forms of speech and to use the Polanyi's term "told". Explicit knowledge manifests itself in forms of speech, written documents, mathematical formulas, graphs, etc. On the other hand, TK is the knowledge we cannot easily articulate into words or "tell". Its primary source is experience as stated by Bratianu \& Orzea, (2010) and in many ways requires a conscious effort to understand its significance and invest in approaches that nurture its transfer, sharing and use within an organization.

As mentioned earlier, there has been an ongoing debate within the knowledge management community on c onversion from one type of knowledge to the other. More specifically, to what degree can TK be articulated and converted to an explicit form. Researchers like Leonard \& Sensiper, (1998) and Richards, (2000) argue that only part of TK also commonly referred to as articulable TK can be articulated for practical and competitive reasons. On the other hand, researchers like Sternberg, (1995) famously argued that "in theory, tacit knowledge can be verbalised and taught". A thorough investigation into the meaning of TK and characterization of TK constructs into articulable and non-articulable was done by Dampney, Busch, \& Richards, (2002) which provides greater understanding into TK evolution process. They concluded that "Tacit knowledge, at least in practice, encompasses a component that lends itself to eventual articulation" (Dampney, Busch, and Richards 2002). Proper definition and understanding of the
TK within the organization is prerequisite before any measures can be placed to quantify it. This requires raising awareness and analyzing closely the KM practices within an organization. Different types which include, but is not limited to, somatic TK, contingent TK, and collective TK can be present or relevant for an organization. Determination of right type and required levels is only possible when a common understanding of what constitutes TK within an organization is developed. In addition to this, there is no acceptable standard for measuring individual TK that is simple and easy to be applied in different fields (Zhi 2016). Knowledge management is regarded as a nonlinear process with all aspects of the process as strongly non-linear (Bolisani and Bratianu 2018). Consequently, management of TK whose primary source is experience as stated by Bratianu \& Orzea, (2010), is also a non-linear process and thus can only be measured through nonlinear metrics. Therefore, different indicators based on the given context must be defined to measure and manage TK within an organization.

Several methodologies have been proposed over time by researchers to measure individual TK. These include methodological approach by Garcia-Perez \& Mitra, (2007) where they suggest conducting action research for identifying TK stocks, its flows, and enablers within an organization and provide a framework to measure TK within an organization. Ribeiro, (2013) also proposed "total time of working experience per levels of similarity" as a unit for estimating the stock of TK after conducting action research in an industrial plant in Brazil. Any proposed transfer, sharing, conversion and measurement strategy for TK needs to address the given context and relevance of individual past work experience with the tasks the individual is currently performing. In this research, the authors rely on the fundamental concept of TK of "knowing more than what can be told" and will investigate relevant TK for Railways. Next section presents the proposed conceptual framework for TK management within the context of systems integration for the Railways sector.

\section{The conceptual framework for TK management for Railways}

As stated in the introduction, a holistic approach towards TK at systems integration level is required within the Railways system. Development of a conceptual framework will lay down the foundations for structurally identifying and managing the utility of TK in the context of systems integration for the Railways system. The proposed framework is developed after understanding the academic debate on the TK as 
presented above and after compiling the findings of seven interviews. These interviews were conducted with the experts in network development, quality and safety management departments of the Netherlands Railways also known as Nederlandse Spoorwegen (NS). Furthermore, two experts from the asset management department of the ProRail, which the rail-infrastructure company of the Netherlands, were also interviewed. During these interviews three fundamental questions were discussed which are as follows:

- How do NS and ProRail share the lessons learned to all the relevant stakeholders after the investigation is complete on an incident like for example an accident?

- What is your reflection on the adequacy of explicit and tacit knowledge management present within your organization related to the functional and operational aspect of the system?

- What is your reflection (based on your experience) on key KPI's that are needed for successful integration of new rolling stock in the current rail infrastructure?

The case made in this paper after incorporating the findings from the brief literature review and conducted interviews is that four fundamental defining features need to be explored for adequate TK management for Railways. These features are context, TK focus, TK constructs and TK embedding as shown in Figure 1.

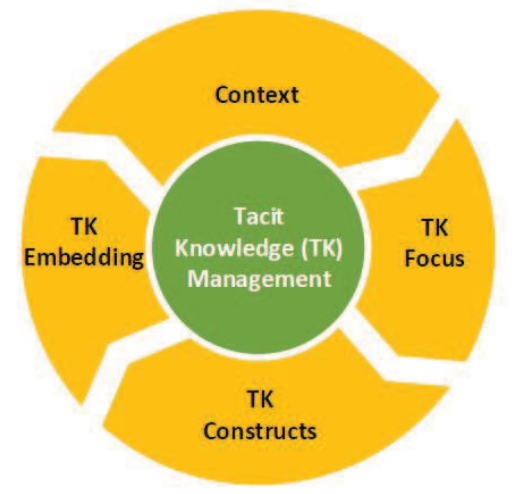

Fig. 1. The conceptual framework for tacit knowledge management
Each of these features is briefly explained and a Railway's specific example is presented in this section.

\subsection{Context}

Proper management of TK requires a clear understanding of the organizational context. This was demonstrated by Evangelista \& Hau, (2009) where they investigated the role of different organizational factors such as management commitment, teamwork, relationship strength and cultural distance on the acquisition of explicit and tacit know-how. Their results indicate that different factors have different effects on explicit and tacit learning and acquisition of each requires different strategies.

An organizational context possesses a lot of knowledge within itself and its proper understanding is fundamental to the accurate definition and proficient management of TK. Besides this, Holste \& Fields, (2010) also stated deficiency of applying context-specific TK in other contexts among other difficulties that may hinder TK sharing. Therefore, clear understanding and boundaries of the context are essential for proper identification and management of TK. The underlying problem needs to be realized by working together with all the relevant stakeholders and an understanding must be developed for the expected desired solution. It is worth mentioning that a problem can be of critical importance in one context and of not that significant in another context. For example, proper functioning of a toilet in a passenger train can be of critical importance in the context of customer satisfaction but of not that significant in the context of technical installation. Thus, the approach towards the management of TK varies depending on the context at hand.

\subsubsection{Context for Railways}

As mentioned in the introduction there is a need for a holistic approach towards TK at systems integration level within the Railways system. The evident next question is then what is a system and what is the systems integration level specifically for Railways? The system as defined by ISO/IEC/IEEE 15288 is a "combination of interacting elements organized to achieve one or more stated purposes". Rajabalinejad, (2018) in his previous work on incorporating safety into design process presented three main blocks namely system, environment, and people that 
must be considered in every design or safety analysis process as shown in Figure 2.

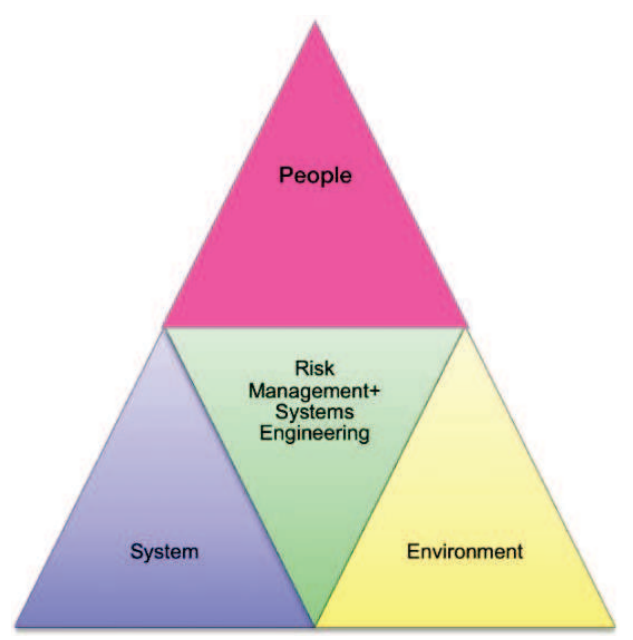

Fig. 2. Three main elements for s ystem design and system safety by (Rajabalinejad 2018a)

Besides this, the purpose of systems integration as defined by ISO/IEC/IEEE 15288 is to "synthesize a set of system elements into a realized system (product or service) that satisfies system requirements, architecture, and design". By extending the system description presented by Rajabalinejad, (2018) to systems integration level and analyzing integration between system, environment, and people for not just system safety but also for system quality, asset management adequately provides the system description for Railways system in the context of systems integration.

The systems of interest for systems integration are then SMS, QMS, and AMS. Integration between these systems through effective and functional interfaces is prerequisite for improving the current performance of the Railways. Similarly, the relevant people for systems integration are project managers, safety managers, implementation engineers, quality managers, system engineers, asset managers, maintenance engineers, etc. within the Railway company. For proper TK management determination of the main tasks and key responsibilities of the stated stakeholders holds critical importance. These key stakeholders with the help from experts in the field of knowledge management can identify and prioritize different types of TK relevant for systems integration. Besides this, key stakeholders can also help in establishing appropriate strategies for managing the determined TK types within their organization. For instance, the conductors of the train possess immense TK about people 's behavior and use of the train. Utilization of this TK is key to improved performance must be incorporated in, for example, the decision-making process of the train operations and designing process of new trains. Moreover, the determination of the environment for systems integration implies determining the cooperating and competing systems with the assistance of stated stakeholders. Therefore, determination of the right systems, people and environment for systems integration as presented is the fundamental first step towards adequate TK management.

\subsection{TK focus}

The second aspect may seem self-evident but plays a crucial role in the successful management of TK. TK focus stresses on a clear understanding of the focus of TK in the determined system of interest and fundamental problem related to TK in that system. By properly defining TK and raising awareness, relevant problems around the domain TK in the system of interest can be identified. As mentioned in the state of the art the concept of TK which can be simply put as the knowledge that cannot easily be articulated into words was first presented by Polanyi when he stated, "we know more than we can tell" (Polanyi 1966). If a metaphor of an iceberg is used for a knowledge possessed by an individual, then the tip of the iceberg can be represented as the individual explicit knowledge and the remaining invisible part can be represented as the individual's TK. In addition to this, TK can be characterized into different types depending on the focus and system of interest like for example somatic-limit and collective TK as presented by Collins, (2007). Therefore, for proper management of TK, clear focus on which TK is the most relevant for the given context is very critical.

\subsubsection{TK focus for Railways}

Once the system description is well understood and the context of investigation is well defined the next natural step is to determine the TK focus in the given context. It was realized during the interviews that the TK related to the structural aspect of the systems integration is relatively well known and quite extensively converted to an explicit form. This is manifested in the form of manuals, guidelines, regulations and other related documents within NS and ProRail. Moreover, comprehensive and mostly reliable manuals and maintenance schedules are provided by Original Equipment Manufacturer (OEM) for trains and 
other rail infrastructure. However, TK related to functional and operational aspects of the system is often overlooked.

It was realized in the interviews that in the context of systems integration for the rail-sector industry it is more interesting to manage TK related to functional and operational aspects of the system. This implies setting the focus of TK management on the role of functional and operational aspects more specifically on human factors/ ergonomics and their role on the overall performance of the Railways. Wilson \& Norris, (2005) reviewed the literature on human factor research within Railways and in his later work Wilson, (2014) stressed on the need of a systems approach towards ergonomics by illustrating with an example from Railways. Therefore, by setting the focus on functional and operational aspects of the system specifically on human factors/ergonomics, appropriate TK for systems integration can be realized.

\subsection{TK constructs}

Once the context is clearly defined and the focus of TK is well aligned next aspect is the classification of this TK into articulable and nonarticulable constructs. An extensive characterization of generic TK constructs has been performed by Dampney et al., (2002), where they state that by adding context, sense, and meaning to data our minds evolve through an unknown process from tacit inarticulable to the explicit and articulable understanding of it. The developed knowledge constructs can then lay the foundations for embedding the identified TK into the current knowledge management system of the organization. The underlying proposition is that articulable TK construct can be converted to an explicit form with proper management approach and non-articulable TK construct is by nature something which cannot be converted to an explicit form and therefore requires different management approach for its adequate utilization in the performance improvement of the system. Furthermore, this construction also assists in managing the levels of uncertainty on the presence of TK within a given context and its relevance for the defined focus at hand.

\subsubsection{TK constructs for Railways}

Construction of knowledge constructs for TK more specifically into articulable and nonarticulable is a challenging task. As stated in the conceptual framework Dampney et al., (2002) constructed generic TK constructs. In the context of systems integration, a thorough investigation into various TK constructs present within the Netherlands Railways and ProRail needs to be done. During the interviews, some TK constructs were identified like for example gut feel, teamwork, the way of doing things, intuition, etc. However, a complete in-depth analysis is required to properly characterize them for determined focus and to propose something on a more generic level which will also be the focus of future research.

\subsection{TK embedding}

The embedding of TK into practice and maximization of its utilization in performance improvement requires proper management strategy. Several knowledge-sharing and knowledge-transfer practices have been investigated by the researchers which include An Fengjie, Qiao Fei, \& Chen Xin, (2004) where they analyzed the knowledge sharing process and developed a web-based knowledge sharing platform to support knowledge sharing within an enterprise. Similarly, Wang \& Noe, (2010) developed a framework of knowledge sharing research which highlights aspects like beliefs of knowledge, trust, the cohesiveness that mostly tacit in nature. Emphasis on these aspects is essential for enhanced knowledge sharing and its management within an organization and can also facilitate in enhancing the quality of the decisionmaking process. For example, Brockmann and Anthony, (2002) demonstrated that TK facilitates in enhancing the quality of strategic decisions. Furthermore, research from Z. Wang \& Wang, (2012) concludes that TK sharing significantly affects innovation quality and operational performance something highly valued within the Railways organizations in general and in this case specifically by the NS and ProRail. Thus, appropriate management approach for TK transfer, share and consequently management should be formulated be it in the form of face to face meeting, one on one apprenticeships, serious gaming, web-based knowledge sharing, etc. Next section provides Railway's specific example of TK management using the presented conceptual framework.

\subsubsection{TK embedding for Railways}

Several programs for knowledge management and knowledge sharing on a systems integration level were identified during interviews within NS and ProRail. Most of these platforms aim to facilitate knowledge sharing and knowledge transfer among their workforce and between 
different programs. This include programs that play the role of interface between different levels of the organization that assist in decision making between the strategic and operational level of these organizations. Similarly, some programs are aimed at sharing lessons learned from the implementation department to the line organization on topics as the introduction of new trains. The identified TK knowledge constructs need to be embedded in the stated program to achieve the fundamental goals of these programs. This will not only improve the performance of these individual programs but also enhance the overall performance of the Railways. Appropriate strategies for TK management and sharing as stated in the conceptual framework can be used to facilitate this embedding process.

\section{Discussion}

The paper has attempted to de monstrate the significance of the tacit dimension of knowledge and presented a conceptual framework for its management in the context of systems integration. A clear roadmap is provided to address TK after incorporating the findings acquired from a brief literature review and interviews. Since expertise in knowledge about a subject is contextual defining the context for TK has been presented as the starting point of the investigation. Consequently, the context under study namely systems integration in the railways is briefly stated. It was identified as a result of this investigation that the main area of focus for the TK in systems integration is functional and operational aspects of the system. More specifically, investigation of TK related to human factors/ergonomics on a systems integration level within Railways, with the primary focus to make respective TK more explicit.

Although this doesn't come as a surprise paradoxically enough the required TK focus relevant for systems integration was tacitly known by the interviewees and was made explicit during the interviews. Furthermore, the classification of TK into articulable and non-articulable knowledge constructs was briefly presented that assists in managing the levels of uncertainty by identifying and classifying relevant TK related to systems integration in a systematic way. Besides this, as displayed the proposed knowledge constructs are embedded in theory and the paper recommends embedding the classified TK constructs in practice. Lastly, it is acknowledged that in the context of systems integration a thorough investigation is required for adequate classification of TK into articulable and nonarticulable TK constructs.

\section{Conclusion and future areas of research}

The paper presents at estable conceptual framework for addressing the tacit dimension of knowledge in the context of systems integration for the rail sector. On the practitioners' level, the model can enable policymakers to devise a better knowledge management policy and allocate resources in accordance with the proposed framework. Similarly, on the theoretical level, the model bridges the gap between challenges within the Railways sector such as systems integration and TK. Furthermore, it provides insight into key features that must be addressed for proper TK transfer, sharing, and management in the stated context. Future research stemming from this work is the testing of the framework in a case study and the realization of the extent to which the framework supports the successful systems integration within the Railways sector.

\section{Acknowledgment}

The presented paper was made achievable with the precious data and suggestions provided by the Netherlands Railways and ProRail.

\section{References}

Al-Hawamdeh, Suliman. 2002. "Knowledge Management: Re-Thinking Information Management and Facing the Challenge of Managing Tacit Knowledge. University Singapore: School of Communication and Information Nanyang Technological." 8 (1).

An Fengjie, Qiao Fei, and Chen Xin. 2004.

"Knowledge Sharing and Web-Based

Knowledge-Sharing Platform." IEEE

International Conference on E-Commerce

Technology for Dynamic E-Business, 278-81. https://doi.org/10.1109/CEC-EAST.2004.43.

Bolisani, Ettore, and Constantin Bratianu. 2018.

"Knowledge Strategies." In , 97-115. Springer, Cham. https://doi.org/10.1007/978-3-31960657-6_5.

Bratianu, Constantin, and Ivona Orzea. 2010. "Tacit Knowledge Sharing in Organizational Knowledge Dynamics." Proceedings of the European Conference on Intellectual Capital, no. March: 107-14.

Brockmann, Erich N, and William P Anthony. 2002. "Tacit Knowledge and Strategic Decision Making." Group \& Organization Management 27 (4): 436-55. 
https://doi.org/10.1177/1059601102238356.

Collins, Harry. 2007. "Bicycling on the Moon:

Collective Tacit Knowledge and Somatic-Limit

Tacit Knowledge." Organization Studies 28 (2): 257-62.

https://doi.org/10.1177/0170840606073759.

Dampney, C N G, P Busch, and D Richards. 2002.

"The Meaning of Tacit Knowledge." Australian

Journal of Information Systems, no. Special

Issue: 3-13.

https://doi.org/10.3127/ajis.v10i1.438.

Evangelista, Felicitas, and Le Nguyen Hau. 2009.

"Organizational Context and Knowledge

Acquisition in IJVs: An Empirical Study."

Journal of World Business 44 (1): 63-73.

https://doi.org/10.1016/j.jwb.2008.03.016.

Garcia-Perez, Alexeis, and Amit Mitra. 2007. "Tacit

Knowledge Elicitation and Measurement in

Research Organisations: A Methodological

Approach." Electronic Journal of Knowledge

Management 5 (4): 373-86.

Holste, J. Scott, and Dail Fields. 2010. "Trust and

Tacit Knowledge Sharing and Use." Journal of

Knowledge Management 14 (1): 128-40.

https://doi.org/10.1108/13673271011015615.

Kakabadse, N.K., a. Kouzmin, and a. Kakabadse.

2001. "Fromtacit Knowledge to

Knowledgemanagement: Leveraging Invisible

Assets." Knowledge and ProcessManagement 8

(3): 137-154. https://doi.org/10.1002/kpm.120.

Leonard, Dorothy, and Sylvia Sensiper. 1998. "The

Role of Tacit Knowledge in Group Innovation."

California Management Review 40 (3): 112-32.

https://doi.org/10.2307/41165946.

Penciuc, Diana, Marie Hélène Abel, and Didier Van

Den Abeele. 2010. "Modelling and Architecture of a Workspace for Tacit Knowledge

Management in Railway Transport."

Proceedings - International Conference on

Tools with Artificial Intelligence, ICTAI 2.

IEEE: $356-57$.

https://doi.org/10.1109/ICTAI.2010.126.

Polanyi, Michael. 1966. The Tacit Dimension.

University of Chicago Press: Chicago, 4.

Rajabalinejad, Mohammad. 2018a. "Incorporation of

Safety into Design Process : A Systems

Engineering Perspective" 20 (3): 1366-68.

. 2018b. "System Integration : Challenges and

Opportunities," 6-11.
Ribeiro, Rodrigo. 2013. "Tacit Knowledge

Management." Phenomenology and the

Cognitive Sciences 12 (2): 337-66.

https://doi.org/10.1007/s11097-011-9251-x.

Richards, Debbie. 2000. "Measuring, Formalising and

Modelling Tacit Knowledge." International

Congress on Intelligent Systems And, 7.

http://scholar.google.com/scholar?hl=en\&btnG

$=$ Search $\& \mathrm{q}=$ intitle:Measuring,++ Formalising $+\mathrm{a}$ nd+Modelling+Tacit+Knowledge\#0.

Siegel, Aron Wolf, and Jan Maarten Schraagen. 2017. "Team Reflection Makes Resilience-Related Knowledge Explicit through Collaborative Sensemaking: Observation Study at a Rail Post." Cognition, Technology and Work 19 (1). Springer London: 127-42.

https://doi.org/10.1007/s10111-016-0400-4.

Sternberg, Robert J. 1995. "Theory and Measurement of Tacit Knowledge as a Part of Practical Intelligence." Zeitschrift Für Psychologie Mit Zeitschrift Für Angewandte Psychologie 203

(4). Germany: Hüthig GmbH \& Co. KG/ Johann Ambrosius Barth Verlag: 319-34.

Wang, Sheng, and Raymond A. Noe. 2010.

"Knowledge Sharing: A Review and Directions for Future Research." Human Resource

Management Review 20 (2). Elsevier Inc.: 115-

31. https://doi.org/10.1016/j.hrmr.2009.10.001.

Wang, Zhining, and Nianxin Wang. 2012.

"Knowledge Sharing, Innovation and Firm

Performance." Expert Systems with

Applications 39 (10). Elsevier Ltd: 8899-8908.

https://doi.org/10.1016/j.eswa.2012.02.017.

Wilson, John R. 2014. "Fundamentals of Systems

Ergonomics/Human Factors." Applied

Ergonomics 45 (1). Elsevier Ltd: 5-13.

https://doi.org/10.1016/j.apergo.2013.03.021.

Wilson, John R., and Beverley J. Norris. 2005. "Rail

Human Factors: Past, Present and Future."

Applied Ergonomics 36 (6 SPEC. ISS.): 649-

60.

https://doi.org/10.1016/j.apergo.2005.07.001.

Zhi, Zeng. 2016. "Review of Individual Tacit

Knowledge Measurement." Humanities and

Social Sciences 4 (6): 135.

https://doi.org/10.11648/j.hss.20160406.11. 